\title{
Starting and resourcing family and internal medicine residency programs as integral mission
}

\author{
Stephen P Merry ${ }^{a}$, Bruce Dahlman ${ }^{b}$, Adam P Sawatsky ${ }^{c}$, Dennis Palmer ${ }^{d}$, Kevin C \\ Shannon ${ }^{\mathrm{e}}$, Thomas D Thacher ${ }^{\mathrm{f}}$ \\ ${ }^{a}$ MD, MPH, Consultant, Department of Family Medicine/Assistant Professor, College of Medicine, Mayo Clinic, \\ United States \\ ${ }^{\mathrm{b}} \mathrm{MD}$, Head, Department of Family Medicine and Community Care, School of Medicine and Health Sciences, \\ Kabarak University, Kenya \\ ${ }^{\mathrm{c}} \mathrm{MD}$, Consultant, Department of Internal Medicine/Assistant Professor, College of Medicine, Mayo Clinic, United \\ States \\ ${ }^{d}$ MD, Program Director, Christian Internal Medicine Specialization Residency, Mbingo Baptist Hospital, Cameroon \\ ${ }^{\mathrm{e}} \mathrm{MD}$, Associate Professor, Departments of Family \& Preventive Medicine, Loma Linda University School of \\ Medicine, United States \\ ${ }^{f}$ MD, Consultant, Family Medicine/Professor of Family Medicine, College of Medicine, Mayo Clinic, United States
}

\section{Introduction}

Graduate medical education is an excellent means of building the capacity of health care systems in low and middle income countries (LMIC) and a growing way for physicians in high income countries to get involved in integral mission - the proclamation and demonstration of the gospel. In November 2015, 67 medical missionaries and academicians met in conjunction with the Global Missions Health Conference (GMHC) in Louisville, Kentucky. A subgroup of 33 physicians and educators held specific discussions about medical residency training programs in LMIC settings and then reviewed the themes they identified with the entire group before sending a summary of those findings for further editing by those educators present as well as those unable to attend who were integrally involved in the development of family medicine and internal medicine residency programs. For ease of discussion, this article will collectively refer to these programs as "medical residency programs."

\section{An Overview of Medical Residency Development in LMIC}

The participants in the expert panel discussion came from varied academic contexts that reflect the variety of the medical residency programs that have started with medical missionary involvement in LMIC the last 50 years. Most of them have been family medicine residency programs. Most of the participants involved in the discussion were of North American origin, some originated and resided on other continents, and most participants had spent years working professionally outside of North America. We reviewed the history of medical residency development, noting the variety of country and health system contexts in which they have developed.

Drs. Calvin Wilson and Warren Heffron chronicled the development of some of the early family medicine programs in a review article in 1994. ${ }^{1}$ They noted that perhaps the first mission hospital general practice residency started in Paraguay in 1951 at Centro Medica Bautista, now a large multi-specialty private medical center. Today,

Nov 2016. Christian Journal for Global Health, 3(2): 151-159. 
there are multiple paradigms in which expats and national educators may be involved in integral mission.

One option is the development of distinctly Christian residency programs like at Kabarak University in the predominantly Christian country of Kenya where one of us, BD (who initiated a related preconference discussion at the GMHC many years ago), is now head of the department of family medicine and director of the residency program that started in 2015. The residents come from Kenya, South Sudan, Burundi, and Democratic Republic of Congo and are recruited with a commitment to work in underserved areas and willingness to work outside of their home culture.

Another model is a medical residency in a church hospital that is not university-based in a predominantly Christian country. Examples are the family medicine program started by Dr. Calvin Wilson at Hospital Vozandes in Quito, Ecuador in 1987 in conjunction with the Catholic University of Cuenca medical school. Graduates of that program have established and have led the Ecuador Academy of Family Physicians. ${ }^{2}$ In Cameroon, the Christian Internal Medicine residency program at Mbingo Baptist Hospital was started and is still directed by one of us, DP, at a hospital owned and operated by the Cameroonian Baptist Convention Health Board.

There are some programs in mixed faith countries with a fair education infrastructure like the Myungsung Family Medicine program starting in Ethiopia. The program at Evangel Hospital in Jos, Nigeria sponsored by the Evangelical Churches of West Africa, was established in 1982 and itself followed the development of family medicine programs at Ogbomosho Baptist Medical Center in 1977 and the program in Eku, Nigeria. ${ }^{1}$ Family medicine has now spread across the country of Nigeria with many residency programs in church and government hospitals. In India, which at one time had some 500 Christian mission hospitals,
Emmanuel Hospital Association which has reopened or taken over some 23 of these mission hospitals, has started a number of family medicine residency programs. ${ }^{2}$

The family medicine residency started in 2009 by Dr. Milad Hanna at Germania Hospital in Aswan, Egypt is an example of a Christian hospital openly training with Christian faculty in a Muslim context. Another of these is the family medicine program started at the CURE International Hospital in Kabul, Afghanistan in 2004. ${ }^{2}$

There are Christian educators working in government family medicine programs with outside funding like those with Society for International Missions (SIM) at Addis Ababa University in Ethiopia $^{2}$ or in a government hospital internal medicine residency program like Drs. Denis Burkitt and Richard Goodgame at Makerere University in Kampala, Uganda and our co-author, TT, who started and led the family medicine department and residency program at Jos University Teaching Hospital in Nigeria for many years before coming to Mayo Clinic.

Finally, there are many Christian physician educators working to establish family medicine programs in closed-access nations (CAN). A complete review of all past and extant programs is beyond the scope of this article; rather this brief overview provides a panorama of the varied terrain in which the many different kinds of programs are growing and flourishing.

\section{Formation}

The steps to formation and accreditation of family medicine residencies have been delineated in other documents. The WHO document on development of family medicine in the eastern Mediterranean region details much of this process and notes that it will be necessarily different in each country. ${ }^{3} \quad$ It may develop through gradual evolution, starting with a prior acceptable model of general practice that may be quite truncated in 
scope from American and European Family Medicine; and then depth and breadth may be added as allowed in that context moving from limited spectrum of outpatient medical care to fullspectrum family medicine - the "pluripotent" physician who capably practices the full breadth of in and outpatient adult medicine, pediatrics, OB/GYN, essential surgery, psychiatry, and has training in community medicine as well. Especially in Africa, this physician provides comprehensive care in a person-centered way while also overseeing the local public health system and community outreach of the hospitals, clinics, and dispensaries within its referral area, functioning as a "consultant to the primary care team" as other cadres provide the majority of first contact and continuity care.

The path to recognition of family medicine is variable. Family medicine may develop suddenly as a specialty as in Nicaragua where full spectrum family medicine started de novo and was recognized by the government from its inception. ${ }^{1}$ In Mauritania, family medicine was first recognized as a specialty by the Ministry of Health (MOH), and then, the first steps were taken to start the program. In Ethiopia, influenced by developments in Kenya and supported by North American academics from University of Toronto and University of Wisconsin (and elsewhere), the $\mathrm{MOH}$ was approached early to seek reassurance that graduates would be provided with jobs and be recognized and paid as specialists on par with other post-graduate specialists. This was successful in launching family medicine training on an apparently sustainable trajectory. In other places, the recognition of family medicine as a specialty by the MOH has been gradual, is still a work in progress, or has not been supported by the $\mathrm{MOH}$ despite best efforts and at least in Cameroon, resulted in the decision to establish an internal medicine with a pediatric training component rather than family medicine residency at Mbingo Hospital.

Nov 2016. Christian Journal for Global Health, 3(2): 151-159.

\section{The Physician Product}

A country's history of primary care and its healthcare needs often determines what the final product of a residency in family medicine or internal medicine will look like. Countries in Africa which lack sufficient general surgery and OB/GYN specialists will necessarily train family medicine specialists to do essential surgery including C-sections. Countries in the former Soviet states will likely presently only accept a final product that resembles the primary care physician of the past - an outpatient-only care provider. In each country and need context, therefore, the curriculum must be adjusted. There will be no "one size fits all" curricular solution or uniform "international standard," though the World Organization of Family Doctors (WONCA) does delineate competencies in the document, "WONCA Global Standards for Post-Graduate Family Medicine Education." ${ }^{4}$ This is not a matter of lowering standards, but of accommodating training to need and medical culture.

\section{Integration or Cooperation of}

\section{Residencies with Local Authorities}

The workshop participants discussed the international movement towards integration of faith-based health care structures in LMIC with the $\mathrm{MOH}$ and national medical education system. They recognized that faith-based organizations have been largely separated from governmental organizations for far too long, functioning in their own "silo" as discussed in a recent Lancet article on faith-based health care in Africa. ${ }^{5}$ While the church hospital and healthcare systems largely pre-date governmental hospitals in Sub-Saharan African, India, and China, participants discussed that we want to avoid "neo-colonialism" by ensuring that we humbly pursue close partnerships with the $\mathrm{MOH}$, and local accrediting agencies while developing and maintaining the spiritual formation aspects of Christian residency training. Regional 
bodies such as the African Christian Health Associations Platform (http://www.africachap.org) have worked to establish such relationships with ministries of health. Such partnerships may require years of patient intentionality to build mutual trust and collegiality. Establishment de novo of a single curriculum and standards used by all residencies which are based on American residency training standards would be unwise and unrealistic and would result in inadequately trained physicians to handle the very different spectrum of disease in LMIC. Rather, standards should be specific to the location with a large degree of overlap that will allow sharing of curricular elements but will require adaptation to the country and context of that region and will sometimes be dictated by the national accrediting body. Training to "international standards" is an artificial construct in light of the dramatic variation in primary care roles around the world. The items that overlap, including knowledge content, multiple-choice questions, curriculum structure ideas, and many other items, can certainly be usefully shared. During a consultation held in Rustenburg, South Africa in 2009, African programs took the initiative to form a consensus statement regarding the unique character and roles of an African family physician which can guide future curriculum development for African family medicine residencies. ${ }^{6}$

Values-based educational elements are important to include in all residencies whether mission or government hospital in which we as Christian physicians are teaching. This should include putting the needs of the patient first, professionalism, integrity, sanctity of life, stewardship of resources, performance excellence, and lifelong learning. These elements can be taught in many ways even in the CAN environment. Embodiment of these values by the faculty is, of course, paramount. ${ }^{7}$ Organizations like PRIME International (UK) have curricula that encourage holistic care and attention to the emotional and spiritual needs of the patient. PRIME seminars have been successfully held in many contexts including secular universities and countries with suppressed Christian minorities. ${ }^{8}$

Recruitment of high quality residents requires recognition that their future employability depends on recognition of their specialty training by the $\mathrm{MOH}$ and licensing bodies as well as acceptance by the country's medical culture. We believe that working within the constructs of the $\mathrm{MOH}$ will result in programs that are ultimately the most sustainable and which successfully build health care capacity through increasing emphasis on training of family medicine, general internal medicine, and pediatric physicians.

Accreditation of programs will depend on location. Where regional or national accreditation systems exist (e.g., West African College of Physicians), applications or, at a minimum, consultations to these bodies must occur at the beginning of the process of program development to promote long-term collaborative working relationships. In an unusual case, an external accrediting body to provide supervision and quality assurance may be necessary.

\section{Relationship with the National Church}

Sometimes the process of residency development meets difficulty as the national church organization affiliated with the hospital is perceived by hospital medical staff to be more concerned about the product — the revenue generated and the physicians educated to staff their hospitals — than valuing high-quality, educational programs. If the national church's operating expenses are supported in part by hospital revenue, the national church may resist use of those revenues to fund medical education and enable the medical center and residency programs to grow. If the vision for the hospital by the two parties differs with missionaries envisioning progressive development of a tertiary teaching hospital and the national church relying on hospital revenues to fund their operating budget,

Nov 2016. Christian Journal for Global Health, 3(2): 151-159. 
crucial conversations conducted in prayerful humility will be necessary to maintain harmony.

\section{Medical Residencies in PAACS Hospitals}

One of the attractive locations for Christian physician academics to be involved in starting medical residency programs and particularly family medicine are hospitals which have Pan-African Academy of Christian Surgeon (PAACS) residencies in Africa and now in Asia at Memorial Christian Hospital in Bangladesh. ${ }^{9}$ The presence of educational facilities and a tradition of education along with the potential for integrated and cooperative training with the general surgery residents can be synergistic. Collegial relationships formed while training together may lead to general surgeon and family medicine teams being sent out to practice together, preventing burnout for both, and extending the reach of the fully qualified general surgeon. Co-training would provide better surgical training for family medicine residents to handle common operative emergencies (i.e., Csections, trauma stabilization, and where and when appropriate surgical management of acute abdomens - the most common conditions that might require a surgeon to get up at night). This sort of task-sharing of fully trained family medicine physician with a fully qualified surgeon with whom they train and with whom they would be sent out would naturally build on the historic model of such task-sharing that has been happening in mission and government hospitals for many decades. ${ }^{10}$ The discussion of the group did not express an expectation of PAACS to take on additional administrative responsibilities for these family medicine residencies, but simply to benefit from the potential synergies resulting from their presence alongside their general surgery residencies.

In November 2015, the groundwork for creating a Christian Academy of African Physicians (CAAP) began at Loma Linda University (LLU) the Christian medical university in California that functions as the residency accrediting body for
PAACS. One of us, KS, has secured administrative support from LLU administration to work with African family medicine and internal medicine academics and several of the authors of this review to develop CAAP to fulfill many of the same functions as PAACS for primary care training programs in Africa with potential expansion to Asia. We anticipate such an organization would support programs in many hospitals where PAACS programs presently exist.

\section{Resourcing the Residencies}

Medical residencies must be resourced in order to develop. A commission in Christian Medical Dental Association (CMDA) would have the advantages of ready name recognition and resources, but the obvious disadvantage of dangerous linkage to Christianity for those working in CAN. Therefore, the structure and tools which we envision being developed will need to be sensitive in content and hotlinks to any Christian organizations that could endanger our colleagues in these settings and yet contain materials which would be uniquely helpful in developing residency programs that produce not just high quality physicians, but disciple physician Christians. We envision a website that provides a wide variety of faith-neutral resources for residency development yet reflects the Christian world view implicitly rather than explicitly and a second website at CMDA that would provide discipleship materials for residencies.

\section{How Educators From High Income \\ Countries Can Assist In LMIC CAN}

In His Image (IHI) Family Medicine Residency Program in Tulsa, Oklahoma provides an exemplary model of how to be involved in residency development from their long experience developing eight residency programs in restricted or CAN. The process of starting a program begins for IHI when they receive a request for a consult. They 
are invited and go in as guests to see who is involved and assess the needs as well as the desire and intent of the government or university - an inside out process rather than going in to suggest a particular product. They don't commit long term until they see that developing a family medicine residency in that location will succeed based on the core resources or assets that are available to meet the needs. Sometimes the formal invitation by the government for IHI to be involved has taken time and followed the government or university's observation of medical education courses in which the scope of family medicine has been demonstrated (i.e., an Advance Life Support in Obstetrics course or other CME courses) before an invitation was extended. They recognize they are wanted some places and not others. They rely on God's sovereignty and the Spirit's calling, knowing that it is God who opens and closes doors. Like IHI, Medical Education International (MEI) desires to partner with nationals and likewise go only when invited.

\section{Specialty Faculty}

A core of long-term faculty is the foundation that provides stability to a residency program. Participants didn't view faculty retention as a significant obstacle to long-term success and noted that most faculty members are recruited from program graduates. In addition to the core faculty, specialists teaching in the residency are important to bring expertise to medical training. Predictably timed visits of national or expatriate specialists who commit to coming at a particular time each year to teach a particular portion of the curriculum is optimal. On years that specialists can't be present, they will ideally be responsible for finding a samespecialty colleague to cover their "slot," assuring that the LMIC medical residency program is dependably resourced in that part of the curriculum. An online recruitment tool that lists opportunities searchable by specialty, country, and perhaps dates is needed to facilitate international networking of LMIC residencies with specialists looking to use their professional skills in this capacity. Some curricular components can be taught by visiting faculty in workshops including locally appropriate life-support courses. "Advanced" courses may be at an inappropriate level for the resources available and the prior training of the participants and so should include some of the basic elements as well (e.g., BLS should be taught before ACLS or ATLS). A comprehensive emergency skills course very appropriate for use in LMIC, taught to a health care team at their own facility, called Comprehensive Advanced Life Support (CALS) Essentials, will soon become available from CALS (www.calsprogram.org). The comprehensive CALS manual is available in Africa for mobile use on smart phone or tablet as part of the Digital African Health Library developed by co-author BD (www.digitalhealthlibrary.net).

\section{Timeframe}

Development of a sustainable program with national faculty requires a long-term vision of 15 or more years to train high-quality generalists in critical thinking, to teach curriculum development and resident evaluation, and the rest of what comprises an excellent residency program. The principles of adult learning, observation and evaluation, patient-centered clinical methods, and research mentoring and facilitation are important aspects of faculty development that appear, in the experience of this workshop's participants, to be often missing in national faculty experiences due to different approaches to education in LMIC as compared to medical education in the United States. Excellent faculty development opportunities exist in most areas through regional FAIMER institutes (www.faimer.org) and with regional health profession educators associations, such as the South African Association of Health Educators (SAAHE) (http://saahe.org.za) or the EAHPEA in Eastern 
Africa, and participation in these should be encouraged to build capacity in these countries (https://sites.google.com/site/eamededucators).

\section{Integral Mission}

Participants agreed that integral mission must be the goal of Christian residency programs. Postgraduate medical education is best done in the context of long-term clinical mentoring relationships for residents by and with faculty over the 3-4 year curriculum and is a great opportunity for spiritual discipleship. Christian physician discipleship tools, such as provided by CMDA, ICMDA and organizations like PRIME International (www.prime-international.org) and mentoring relationships linking physicians as is found on the GMHC website under the prior name of Medical Education Missions (www.medicalmissions.com/network/education) will be essential in resourcing faculty to be involved in discipleship in these residency programs. The presently available mentoring Medical Education Missions website has received few "hits" since inception. Reasons for this may be that the resources provided do not meet felt needs, or the site may be at an unknown location that is not easily located by individuals with those needs, or the site has not provided enough information about what the user (prospective mentor or mentee) could expect. A formal needs assessment would clarify the situation. A residency development website located within GMHC or at CMDA as part of a larger educational commission searchable by country, residency type, specialty needed, and resources needed (education, research assistance, patient care) could provide networking assistance to connect Christian healthcare professionals with education institutions, including medical residencies worldwide. Strategic opportunities in CAN should be emphasized.

\section{Conclusion}

The preconference workshop participants concluded their session by sharing their vision for the outcome of the meeting to resource the development of high quality medical residency programs in response to God's call and in His timing in order to build His Kingdom. Participants suggested doing this through the creation of two websites which can resource residencies: one acceptable to access in CAN with additional human resource networking and another providing Christcentered educational resources perhaps hosted by CMDA. These programs exist in varied contexts thus serving the variety of needs for capacity building in these countries. The final physician product of these residencies will depend on the degree to which a progressive view of a broadly trained physician capable of performing outpatient primary care as well as inpatient care and essential surgery has developed in each individual country. But, participants largely agreed that integration and cooperation with the country's $\mathrm{MOH}$ is essential for sustainable health development, recognizing that parallel mission and government health systems have been fairly labeled "neo-colonialism", making such integration timely and imperative. Recognition of family medicine as a specialty by the $\mathrm{MOH}$ prior to starting training will prepare graduates of those programs for success in the country's job market and health systems leadership. Recognition by the national church of the unique needs of their mission hospitals' educational programs to control their revenue in order to fund their programs' growth and development exemplifies the common wisdom to provide authority and resources where responsibility for good outcomes is expected. Co-training of general surgeons and medical residents who can provide essential surgical call coverage may lead to on-going synergies. Medical and surgical subspecialists are essential in family and internal medicine residencies to provide the depth of instruction residents need to develop as excellent clinicians. Dependable 
scheduling of their specialty instruction allows residency program directors to assure inclusion of their content in the residency curriculum. In summary, participants agreed that teaching in medical residency programs in LMIC present excellent opportunities for national and expat Christian physician educators interested in integral mission.

\section{References}

1. Wilson C, Heffron W. Christian hospitals as family practice educational resources. Educ Res Methods.1994;26(9):571-5.

2. Heffron W, Jenkins C. Serving overseas: volunteer teaching [Internet]. Consultant. 2011;51(8): [cited 2016 Aug 25] Available from: http://www.consultant360.com/content/servingoverseas-volunteer-teaching

3. World Health Organization, Regional Office for the Eastern Mediterranean. Conceptual and strategic approach to family practice: towards universal health coverage through family practice in the Eastern Mediterranean Region . 2014. [cited 2016 Aug 25] Available from: http://applications.emro.who.int/dsaf/EMROPUB 2014_EN 1783.pdf?ua=1

4. WONCA Working Party on Education. WONCA Global Standards for Postgraduate Family Medicine Education [Internet]. 2013 June. [Cited 2016 August 25] Available from: http://www.globalfamilydoctor.com/site/DefaultSi te/filesystem/documents/Groups/Education/WON
CA\%20ME\%20stds_edit\%20for\%20web_250714 .pdf

5. Olivier J, Tsimpo C, Gemignani R, Shojo M, Coulombe H, Dimmock F, et al. Understanding the roles of faith-based health-care providers in Africa: review of the evidence with a focus on magnitude, reach, cost, and satisfaction. Lancet. 2015;386(10005):1765-75. http//dx.doi.org/10.1016/S0140-6736(15)60251-3

6. Mash R, Reid S. Statement of consensus on family medicine in Africa. Afr J Prm Health Care Fam Med. 2010;2(1):Art.\#151. http://dx.doi.org/10.4102/ phcfm.v2i1.151

7. Jones W, Hanson J, Longacre J. An intentional modeling process to teach professional behavior: students' clinical observations of preceptors. Teach Learn Med. 2010;16:264-9. http://dx.doi.org/10.1207/s15328015tlm1603_8

8. PRIME-international.org [Internet]. East Sussex, UK; PRIME [cited 2016 September 19].

Available from: http://www.primeinternational.org/.

9. Pollock JD, Love TP, Steffe BC, Thompson DC, Mellinger J, Haisch C. Is it possible to train surgeons for rural Africa? A report of a successful international program. World J Surg.

2011;35(2):493-9. http//dx.doi.org/10.1007/s00268-010-0936-z

10. Merry SP. Re: Is it possible to train surgeons for rural Africa? A report of a successful international program. World J Surg. 2011;35(9):2172-4. [author reply 2175-6] http://dx.doi.org/10.1007/s00268-011-1154-z

Competing Interests: None declared.

Correspondence: Stephen P Merry, Mayo Clinic, United States. merry.stephen@mayo.edu Bruce Dahlman, Kabarak University, Kenya. bruce.dahlman@aimint.org Adam P Sawatsky, Mayo Clinic, United States. Sawatsky.Adam@mayo.edu Dennis Palmer, Mbingo Baptist Hospital, Cameroon. palmerdd47@gmail.com Kevin C. Shannon, Loma Linda University School of Medicine, United States. kshannon@llu.edu Thomas D. Thacher, Mayo Clinic, United States. thacher.thomas@mayo.edu 
Cite this article as: Merry SP, Dahlman B, Sawatsky AP, Palmer D, Shannon KC, Thacher TD. Starting and Resourcing Family and Internal Medicine Residency Programs as Integral Mission. Christian Journal for Global Health (Nov 2016), 3(2):51-59.

C Merry SP, Dahlman B, Sawatsky AP, Palmer D, Shannon KC, Thacher TD. This is an open-access article distributed under the terms of the Creative Commons Attribution License, which permits unrestricted use, distribution, and reproduction in any medium, provided the original author and source are properly cited. To view a copy of the license, visit http://creativecommons.org/licenses/by/4.0/

www.cjgh.org

Nov 2016. Christian Journal for Global Health, 3(2): 151-159. 